Article

\title{
Hidden Imprints of Minor Merging in Early-Type Galaxies: Inner Polar Rings and Inclined Large-Scale Gaseous Disks In S0s
}

\author{
Olga Sil'chenko \\ Received: 15 October 2015; Accepted: 16 December 2015; Published: 24 December 2015 \\ Academic Editors: José Alfonso López Aguerri, Enrichetta Iodice and Alexei Moiseev \\ Sternberg Astronomical Institute, Lomonosov Moscow State University, University av. 13, Moscow 119991, \\ Russia; olga@sai.msu.su
}

\begin{abstract}
I discuss my latest observational data and ideas about decoupled gaseous subsystems in nearby lenticular galaxies. As an extreme case of inclined gaseous disks, I demonstrate a sample of inner polar disks, derive their incidence, about 10\% among the volume-limited nearby S0 galaxies, and discuss their origin. However, large-scale decoupled gaseous disks at intermediate inclinations are also a rather common phenomenon among the field S0 galaxies. I suggest that the geometry of outer gas accretion and the final morphology of the galaxy may be tightly related: inclined gas infall may prevent star formation in the accreted disk and force the disk galaxy to be a lenticular.
\end{abstract}

Keywords: lenticular galaxies; evolution of galaxies; galaxies: interstellar matter; galaxies: kinematics and dynamics

PACS: 98.52.Lp, 98.58.Ay, 98.62.Ai, 98.62.Hr, 98.62.Lv

\section{Introduction}

Minor merging, or gas accretion from outside, is now thought to be the main driver of evolution of disk galaxies [1]. Neither stable continuous star formation in disks of spirals, nor the observed chemical evolution of their stellar populations can be explained without admission of intense gas supply from outside [2-4]. However, direct observational findings of such events are rather rare though we would suggest them to happen daily. Perhaps, it would be easier to search for observational signatures of gas-rich minor merging just in early-type disk galaxies, S0s, where their own gas confined to the main galactic disks does not prevent rather long-lived kinematical misalignments between the stellar disks and the accreted gaseous subsystems to exist.

Unlike the common prejudices provoked by the absence of large-scale star formation in the S0 disks, just luminous S0s rather often possess cool and ionized gas. According to the study of the volume-limited sample by Welch and Sage, about $60 \%$ of nearby S0s possess cool, neutral and/or molecular, gas [5]. A slightly lower estimate, $40 \%$, is given for the united, E+S0, volume-limited sample of early-type galaxies in the ATLAS-3D survey [6]. The gas in S0s often reveals itself as regularly rotating ionized-gas disk (51\% of all ETG [7]), though it is mostly ionized not by young massive stars [8]. All this gas seems to be accreted in recent events from outside. It is implied by frequent misalignments between the rotation axes of the stellar and gaseous components in S0s. Bertola [9] proposed a simple model of possibly isotropic gas accretion, and if a particular event had a random direction of gas infall, then just the half of all accreted gas systems would show visible gas counterrotation with respect to the stellar component if projected onto the line of nodes. Early estimates by [10] gave $24 \%$ of all S0s with the ionized gas demonstrating visible gas counterrotation when the spectrograph slit was aligned with the disk isophote major axis. It means that about $50 \%$ 
of all gaseous disks in nearby S0s have an external origin. However, recently, it became clear that the frequency of accreted gaseous subsystems in S0s related strongly to their environment density. Comparing the Virgo cluster members with the S0s in the field, the ATLAS-3D team has claimed that the misaligned gaseous systems are 4 times more frequent in the field S0s than in the cluster members [7]. And when we have studied the sample of strictly isolated nearby S0s, we have found that half of all ionized-gas disks in the isolated S0s counterrotates the stars when observing with the long-slit spectrographs along the disk isophote major axes [11]. So in the extremely rarified environments all the gaseous subsystems in $\mathrm{S} 0$ galaxies are accreted from outside. How recently? It is a separate and very difficult question.

In this contribution I will review such particularly interesting phenomena as circumnuclear polar gaseous disks and large-scale inclined gaseous disks in lenticular galaxies. Their geometry is interesting not only from the point of their origin, but the dynamical view of such systems is also very important to restrict the timescales of possible gas acquisition. If we associate the emergence of outer high-momentum gas accretion sources with the epoch of thin stellar disk formation (in our Galaxy the thin disk started its formation some 8 Gyr ago), then, I think, the observed accretion events may be distributed over the past $8 \mathrm{Gyr}$, within the redshift range of $z=0-1$, and they may be an important factor of the disk galaxy secular evolution.

\section{Inner Polar Rings}

\subsection{How Do We Search for Inner Polar Disks}

The first time when a gas rotation plane in the center of a galaxy was found to stay at the right angle to the rotation plane of the stellar component was probably the case of NGC 2217 where Bettoni with co-authors [12] studied stellar and gas kinematics by means of long-slit spectroscopy. The galaxy is a bona fide barred lenticular, and the circumnuclear polar disk being a strongly warped central part of the regularly rotating large-scale ionized-gas disk settles in one of the main planes of the central triaxial potential, orthogonally to the largest bar axis. Soon after that we started the first survey of nearby galaxies by means of integral-field spectroscopy-the Multi-Pupil Fiber Spectrograph of the Russian $6 \mathrm{~m}$ telescope began to operate in its effective fiber-lens mode. As a result of our study of the central stellar and gaseous kinematics in the nearby disk galaxies, we found distinct inner polar ionized-gas disks in NGC 2841 [13] and in NGC 7217 [14]—early-type spiral galaxies with regular large-scale coplanar, corotating gaseous disks. It is interesting that both spirals are not classified as barred galaxies though some doubts about their strict axisymmetry are implied by their isophote characteristics.

The benefits of panoramic spectroscopy are crucial for effective quantitative diagnostics of the polar orientations of gas rotation planes: by having a two-dimensional distribution of line-of-sight velocities, we can fit it with a circular rotation model and so determine two angles defining completely the rotation plane orientation in space: the inclination to the line of sight and the line-of-nodes position angle on the sky. Only knowing both of them, we can compare spatial orientations of the stellar and gaseous disks and to calculate their mutual inclination:

$$
\cos \Delta i= \pm \cos \left(P A_{s}-P A_{g}\right) \sin i_{s} \sin i_{g}+\cos i_{s} \cos i_{g}
$$

This equation has two possible solutions because generally we do not know what half of a galactic disk is closer to us [15]. If one of the solutions gives the orientation close to the polar one, we prefer it because the polar rotation is much more long-lived than the inclined one. Figure 1 presents an example of the inner polar disk in a lenticular galaxy: it is NGC 2962 observed by Alexei Moiseev in 2007 at the Russian 6-m telescope with the Multi-Pupil Fiber Spectrograph (MPFS). Though the kinematical major axes (the directions of the maximum line-of-sight velocity gradients) of the stellar and ionized-gas components are nearly orthogonal, the true mutual inclination between the rotation 
planes of the stars and the ionized gas is either $84^{\circ}$ or $60^{\circ}$; in any case, the central ionized-gas disk is strongly inclined to the main galactic plane, and we classify this configuration as an inner polar disk.
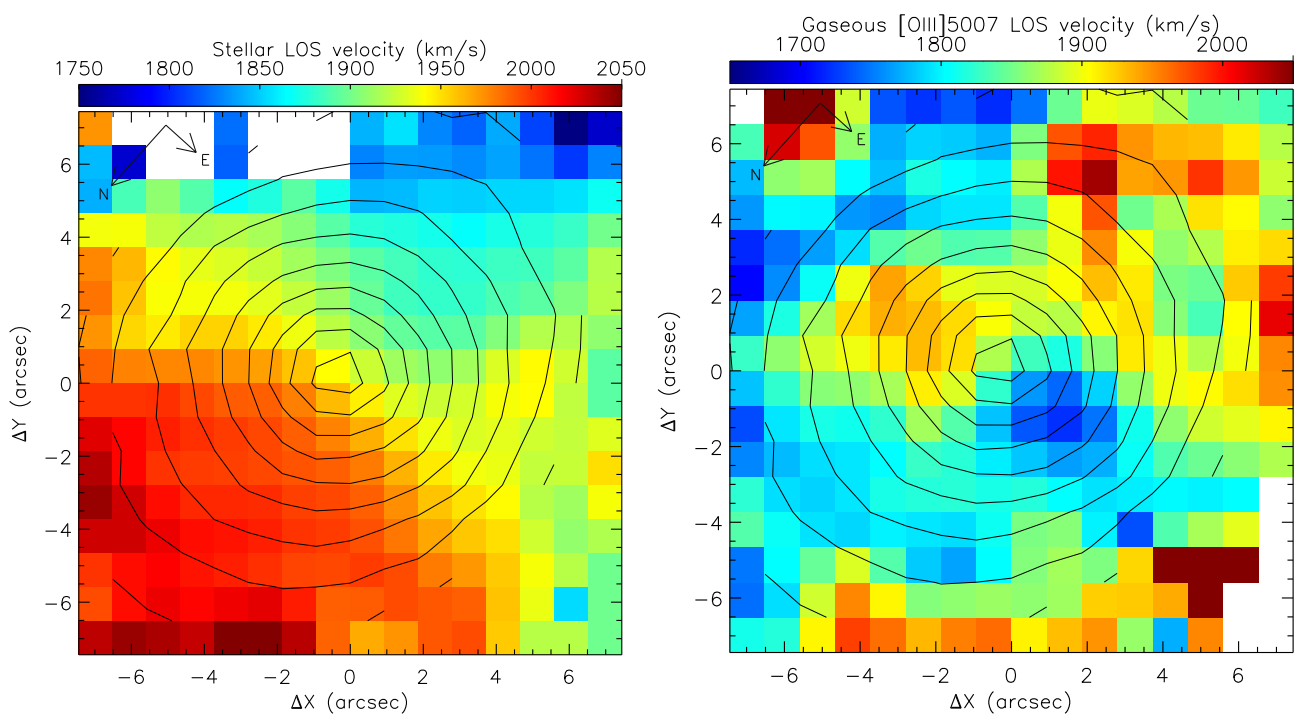

Figure 1. The stellar and ionized-gas line-of-sight velocity fields for the central part of the gas-rich S0 galaxy NGC 2962 obtained with the IFU Multi-Pupil Fiber Spectrograph (MPFS) of the Russian 6m telescope (A. V. Moiseev, private communication); the broad-band green isophotes are superimposed. Despite the visible orthogonality of the rotation planes, the true mutual inclination between the stars and the gas rotation axes can be either $84^{\circ}$ or $60^{\circ}$.

The process of searching for the inner polar disks started, and after a few years the list of the cases found included 17 galaxies [16], among which there were only $7 \mathrm{~S} 0 \mathrm{~s}$ and only 3 barred galaxies; the latest morphological type of the galaxies in this list was Sc. We have applied additional efforts and have observed a sample of S0 galaxies with dust lanes along their minor axes with the Multi-Pupil Fiber Spectrograph (MPFS) of the Russian 6-m telescope; in all 8 S0 galaxies we have found strongly inclined central gaseous disks [17].

\subsection{Properties and Incidence}

Recently, Alexei Moiseev has reviewed the results of a decade of active searches and has published a list of 47 inner polar disks [15]. This number is already sufficient for statistics. We can now state that inner polar disks tend toward early-type disk galaxies: about a half of the list is $\mathrm{S} 0$ galaxies, and only a few are later than $\mathrm{Sb}$. The proportion of barred galaxies among the inner polar disk hosts is equal to the fraction of barred galaxies among all disk galaxies, so we can conclude that inner polar disks do not prefer strong bars though just strong bars might provide a certain triaxial potential for the gas to settle in one of their main planes. The typical radii of the inner polar disks are $0.2-2 \mathrm{kpc}$, and while the inner border is imposed by the spatial resolution of our observations, the outer one has probably the physical meaning. We may suggest that the radius range populated by the inner polar disks is limited by the area of spherical bulge domination. Though Moiseev [15] selected the inner gaseous disks which rotation axes were inclined by only more than $50^{\circ}$ to the stellar rotation axes, the distribution of their mutual inclinations is strongly peaked at $90^{\circ}$, so the disks selected as strongly inclined are indeed polar. We are not surprised: according to many dynamical considerations, just a polar plane is a stable place for gas to stay there for many Gyrs.

However, the review by Alexei Moiseev included a very heterogeneous collection of casual observational findings. The question remains: What can be a frequency of the inner polar disks/rings in the whole ensemble of nearby lenticular galaxies? To address this issue, I have involved the large volume-limited sample of early-type galaxies (ETG) from the survey ATLAS-3D [18]. The sample 
consists of 260 nearby, $D<42 \mathrm{Mpc}$, ETGs, and the majority of them, 200 galaxies, are early-type disk galaxies-lenticulars. I have retrieved the freshest, of 2007-2008, raw SAURON data for 150 S0s from the Isaac Newton Group (ING) Archive (CASU Astronomical Data Centre at the Institute of Astronomy, Cambridge), after their proprietary time has been finished, to analyze the kinematics of the stellar and ionized-gas components (by using the emission line [OIII] $\lambda 5007$ ) for the central parts of these galaxies, with the final aim to search for new cases of the inner polar disks. In this sample I have found 7 new cases of strongly inclined inner gaseous disks. With the inner polar disks mentioned by [15], there are 20 inner polar rings in the $\mathrm{S} 0$ galaxies of the whole ATLAS-3D survey sample, among 200 nearby S0s (150 S0s observed in 2007-2008 plus 50 more S0s from the early SAURON survey). So, the frequency of the inner polar disks/rings in nearby S0s is at least $10 \%$. It is much higher than the frequency of large-scale polar rings, which look a rather exotic phenomenon, $<1 \%[19,20]$.

\subsection{Origin}

The origin of the inner polar disks remains to be a topic of discussion. Partly this situation is supported by a huge variety of morphologies of the outer gaseous disks in the host galaxies of the inner polar disks. For example, the spiral galaxies with the inner polar disks NGC 2841 and NGC 7217 have large-scale abundant neutral-hydrogen disks which are perfectly coplanar to their stellar disks and rotates quite regularly. It is unclear how the polar gas could penetrate into the centers of the galaxies. As for lenticular galaxies with the inner polar disks, they are often gas-rich, but the outer neutral-hydrogen disks are sometimes obviously related and sometimes apparently unrelated to the inner polar gas. After the ATLAS-3D team had undertaken sensitive and rather high-resolution survey of their full sample in the $21 \mathrm{~cm}$ line [6], among their results we could see polar large-scale neutral hydrogen, evidently accreted from the neighbouring irregular galaxy in the S0 NGC 7280 where we found earlier polar rotation of the very central, $R<7^{\prime \prime}$, ionized gas [21]. However, we find also quite regular large-scale massive HI disk in NGC 2962 which continues out the large-scale stellar disk [6].

When there exists evident accretion of the outer cool gas with the polar orbital momentum, the origin of an inner polar disk is quite clear. However, when the outer gas is confined to the main galactic plane, do the possibilities exist to relate it with the inner polar gas? Quite curiously, even two theoretical mechanisms have been published up to now which allow to relate inner polar and outer in-plane gas. They differ in what to consider as a primary gaseous component. Van Albada et al. [22] considered the behaviour of the initially polar gas inside the tumbling, rigidly rotating triaxial stellar body. They have shown that the outer parts of this polar gaseous disk are forced to warp in such a way that the outermost of them lie in the main plane and counterrotate the stellar component. Curiously, Friedli and Benz [23] started their consideration from the case of counterrotating large-scale gaseous disk quite coplanar to the stellar one; and if a strong bar developped in the center of a model galaxy, the counterrotating gas, inflowing into the center, left the galactic plane and stayed for a billion year in a strongly inclined, compact circumnuclear gaseous disk. Both models, despite the difference of their dynamics, implied the presence of triaxial stellar structures (bars?) and of counterrotating outer gas.

The present statistics of the inner polar disk host properties do not match the predictions of these models. Though we have found a few cases of the inner polar disks which warp in the galaxy outer parts into counterrotating large-scale gaseous disks (for example, it is the case of NGC 7280 [24] and NGC 7742 [25]), it is not the rule. There are much more cases of the outer regularly corotating gaseous disks. Similarly, there is no obligatory presence of a bar in any galaxy with an inner polar disk-the fraction of barred hosts of the inner polar disks is quite the same as the fraction of barred galaxies among all disk galaxies [15]. We then must suggest that inner polar disks have to be mostly formed by accretion of the outer gas with a corresponding (polar) orbital moment. It may be tidal gas acquisition from a neighboring comparable-sized galaxy or a minor merger of a small satellite from a highly inclined orbit. For the particular case of NGC 7217 we have selected a minor merging configuration producing its inner polar disk by using the dynamical simulations of the GALMER 
database [26]: if an initially gas-free giant $\mathrm{S} 0$ galaxy devours a gas-rich satellite ten times less massive than the main galaxy, taken from a highly inclined orbit with retrograde orbital momentum, the stable quasi-polar inner gaseous disk with a radius of about $1 \mathrm{kpc}$ forms [27]. Under such circumstances the outer in-plane gas must be accreted later than the inner polar gas, because the gas is a collisional system from a dynamical point of view, and the polar-momentum gas cannot reach the center if interacts with the outer in-plane one. Therefore, if we see two gaseous disks with different sizes and different orientations of their rotation axes in one galaxy-we see the consequences of two different minor-merger or accretion events. Sometimes, the latest of those can transform a lenticular galaxy into a spiral one, as it had happened with NGC 7217.

\section{Large-Scale Inclined Gaseous Disks in Lenticular Galaxies}

If there are no preferred directions of outer gas accretion and if multiple accretion events with isotropic distribution of the infall directions are a common thing for lenticular galaxies, we must see not only strictly polar disks - we must see also the gaseous disks in S0 galaxies characterized by all intermediate inclinations. Their lifetimes are now a point of discussion because their inevitable settlement into the main plane of a galaxy during one free-fall time (about $1 \mathrm{Gyr}$ ) may be delayed by long-lived, continuous accretion process-from a strictly fixed cosmological filament or from extended tidal tails formed during merger event [28]. Taking into account timescales of these reverse infall processes, of a few Gyr, in [28] a visibility of inclined gaseous rings during 3-6 Gyr is predicted. If so, the large-scale gaseous disks/rings inclined by intermediate angles may not be so rare in lenticular galaxies. Indeed, we see such inclined gaseous disks in some S0 galaxies in our observations.

We have undertaken detailed studies of several particular cases. For example, in NGC 7743, a strongly barred S0, the ionized gas beyond $1.5 \mathrm{kpc}$ from the center rotates being inclined to the main galactic plane by $34^{\circ} \pm 9^{\circ}$ or $77 \pm 9^{\circ}$ [29] and is excited by shock waves. In NGC 5631 the outer stellar disk is seen perfectly face-on, and so we can unambiguously determine the inclination of the inner rotating stellar-gaseous disk: it is $35^{\circ}$, quite far from the polar orientation [30]. The gas excitation over the whole extension of the inner inclined gaseous disk-we called it here "inner" though it extends up to at least $5 \mathrm{kpc}$ from the center-is also shock-like: the low-excitation forbidden emission line [NII] 26583 is everywhere stronger than $\mathrm{H} \alpha$. Though the current star formation is absent in the accreted gas of NGC 5631, in the narrow radius range we see the stellar component related to it, in projection onto the apparently non-rotating (because face-on) main stellar disk. Perhaps, in NGC 5631 the inclined stellar-gaseous disk was formed by debris of disrupted accreted satellite that provided both the gaseous and the stellar components with inclined momenta simultaneously.

It is an interesting question, about the origin of the stars which are sometimes observed to be coupled with the accreted gaseous component. There are some well-known cases when two counterrotating coplanar stellar disks are observed in $\mathrm{S} 0$ galaxies, and the ionized-gas component rotation is coupled with one of them. In most of these cases-NGC 3593 [31], NGC 4138 [32], NGC 5719 [33], IC 719 [34]—the secondary stellar components related to the counterrotating (so accreted!) gas, look younger than the main stellar disks, and often current star formation is observed in the inner ring zones, implying that probably the secondary stellar component has also formed "in situ". We have our own example of such galaxies, NGC 4191, the kinematical data for which are shown in Figure 2. According to our long-slit spectral data obtained with the reducer SCORPIO-2 [35] of the Russian 6-m telescope, the galaxy exhibits fast rotation of the ionized gas in a rather extended, $R \geq 3 \mathrm{kpc}$, disk while the stellar component counterrotates in the very center and at the outskirts, and demonstrates the apparently flat $v_{l o s}$ profile at intermediate radii. Since the stellar velocity dispersion reveals off-center maxima in this galaxy [36], we conclude that the apparently flat $v_{\text {los }}$ profile is an effect of two co-adding counterrotating stellar components. Recently Coccato et al. [37] have confirmed the presence of two counterrotating stellar disks in NGC 4191 with the high-resolution integral-field spectroscopic data. The galaxy demonstrates a system of 
starforming rings between $R=15^{\prime \prime}-30^{\prime \prime}$, and the study of this galaxy by [37] has revealed a younger age of the secondary stellar disk coupled with the ionized-gas component traced up to $R \approx 60^{\prime \prime}$.
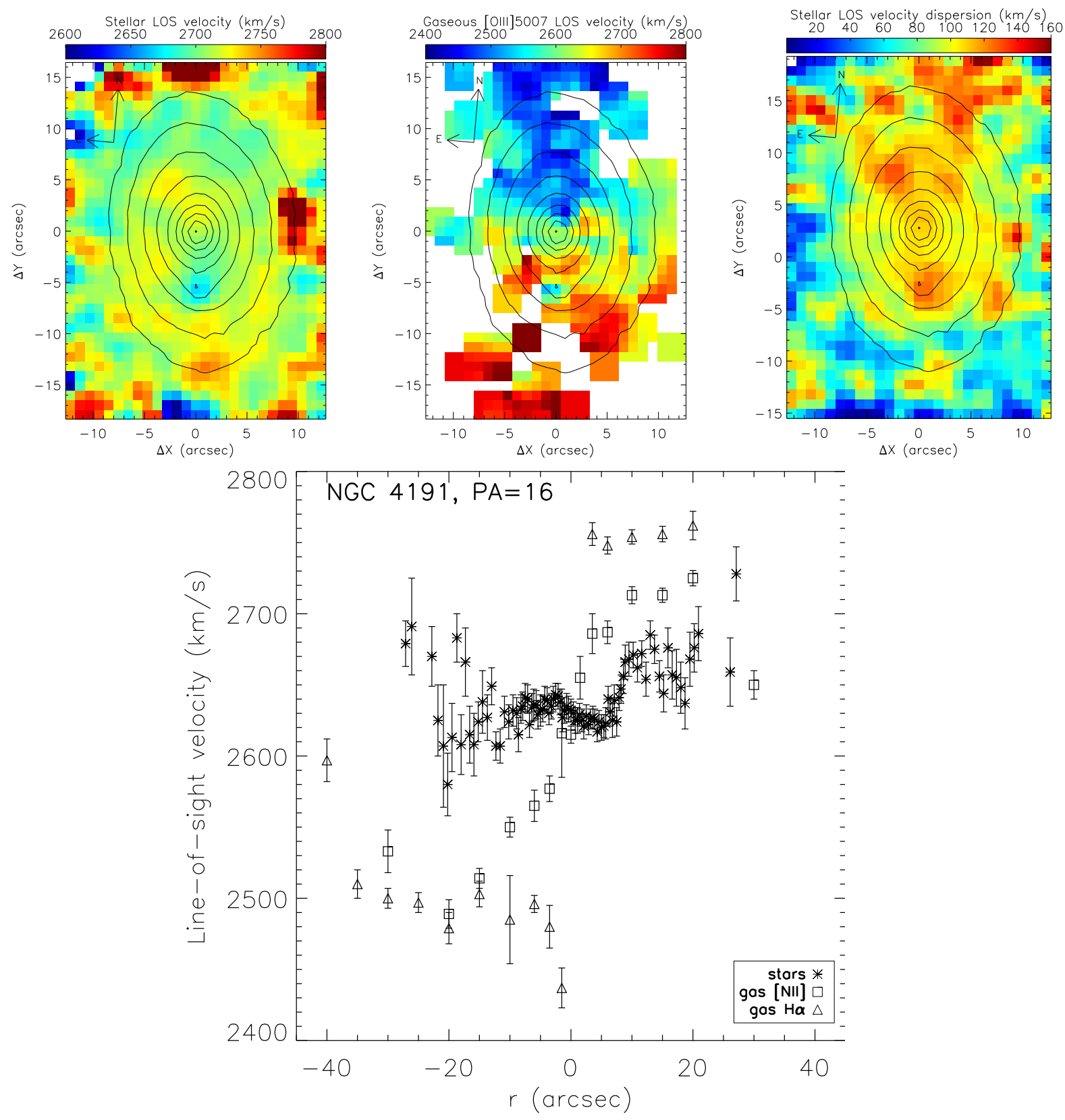

Figure 2. Kinematical data for the S0 galaxy NGC 4191-member of the Virgo cluster. The upper row presents the panoramic spectral data obtained with the IFU SAURON: the LOS velocity field of the stellar component, the ionized-gas ([OIII] $\lambda 5007$ ) LOS velocity field, the map of the stellar velocity dispersion. The bottom plot shows the long-slit cross-section close to the major axis, in $P A=16^{\circ}$, made with the reducer SCORPIO-2 [35] of the Russian 6m telescope in the long-slit mode.

When studying a representative sample of strictly isolated lenticular galaxies for which the outer gas accretion is perhaps a frequent event of their evolution [38], we have noticed that starforming rings where the gas is excited by young stars are common in the counterrotating (and also in corotating) gaseous disks when they look coplanar to the stellar ones. If a gaseous disk is obviously inclined, as a rule its emission-line spectrum reveals shock-like excitation. This 
observation if confirmed by larger statistics may have far-reaching consequences for our ideas about disk galaxy evolution.

\section{Discussion: The Disk Galaxy Evolution Paths}

\subsection{Do Lenticulars form in Clusters?}

The observational result obtained by Butcher and Oemler and summarized in their final paper [39] concerned the difference in content of galaxy clusters between $z=0$ and $z=0.4$ : in nearby rich clusters, like Coma, most galaxies were red, being morphologically ellipticals and lenticulars, while in distant clusters the fraction of blue galaxies rised significantly, up to $25 \%$. They suggested that the effect is due to transformation of spiral galaxies into lenticulars just in cluster environment between $z=0.5$ and $z=0$. When the Hubble Space Telescope (HST) started its high-resolution imaging, the morphological classification, in addition to the photometry, of the galaxies in distant clusters became possible, and the decrease of $\mathrm{S} 0$ fraction and the increase of $\mathrm{S}$ fraction in the clusters at $z=0.4-0.5$ was found in the observational data [40,41]. It looked like we observed the spiral galaxies transformation into S0 "by eye", and just in the cluster environment. The observations of the Butcher-Oemler effect have provoked theoreticians to propose various mechanisms of the spiral transformations into $\mathrm{S} 0$ most of which are specific for the cluster environments.

Historically the first detailed scenario was proposed by Larson et al. [42] just after the first publications about the Butcher-Oemler effect appeared. This scenario included all the necessary state-of-art components of the galaxy evolution known already in the late 70th: short timescales of gas consumption by current star formation in galactic disks, of only a few Gyrs, and so a necessity of external cold gas reservoirs for star formation to proceed during Hubble time; the unavoidable gas-reservoir removing by tidal forces while the cluster collapsed; and even metallicity-age degeneracy allowing lenticulars and ellipticals to have the same colours under quite different ages of the stellar populations. The scenario relates to the class later called "starvation": a spiral galaxy falls into a cluster at $z \approx 0.4$, loses its outer gas envelope immediately, and then consumes the residual gas of the disk by star formation during a few Gyr. After that a S0 arises, without gas, without current star formation, and with a large bulge-to-disk ratio due to the early stop of large-scale disk building. This scenario is still quite valid and competitive with others now $[43,44]$.

Another possibility to transform a spiral into $\mathrm{S} 0$ in the cluster environment is provided by the interaction of the hot intracluster medium with the proper cold gas of the galactic disks. Because of the fast motions of galaxies within the cluster potential (with speeds of several hundreds of kilometres per second), ram pressure of the hot diffuse gas filling the cluster dark halo removes the cold gas from the galactic disks [45]; due to this process a spiral galaxy can be devoid of all its neutral hydrogen for some $10^{8}$ yrs [46]. Since the molecular gas, the immediate fuel for star formation, concentrates in the central parts of spiral galaxies, it may not be expelled but compressed by the hot gas ram pressure and turn into stars with the rate exceeding the usual SFR in spiral galaxies by a factor of 3 at least [47]. So the resulting $\mathrm{S} 0$ is not only lacking the gas but has acquired additional stellar component in its central part to increase the bulge. This mechanism of spiral transformation into $\mathrm{S} 0$ is among the most popular ones.

Among gravitational mechanisms acting in the cluster environment and using to produce a lenticular galaxy from a spiral one, harassment and tidal heating are rather popular. The harassment is pairwise gravitational interaction of two galaxies passing each other at a very high relative velocities, of a few thousand $\mathrm{km} / \mathrm{s}$ [48,49]; several such close encounters would result in gas rotation distortion and its concentration in the galaxy's center, with subsequient violent star formation contributing to the bulge growth. Tidal heating is not a pairwise process but instead reflects the interaction between a spiral galaxy infalling into a cluster and the whole cluster gravitational potential [50]. It is also effective in distorting the large-scale gaseous disk of a spiral galaxy, in turbulizing it, and so depressing star formation in the outer parts of the galactic disks, as opposite 
to the well-known paradigm of disk formation inside-out. Intense star formation in the very center, however, is still possible which would grow the bulge to fit a common opinion of large bulges in lenticular galaxies.

\subsection{Do Lenticulars form in Groups?}

However, recently clusters at $z=0.4$ as a main site of S0 galaxies formation have been put into doubt. Indeed, to make the above mentioned conclusions, it is necessary to study all types of environments, not only clusters. Wilman et al. [51] have selected groups at the same redshift, $z=0.4$, by using optical criteria, so not all of these groups have even hot intragroup medium (not all are detected in X-rays). The fraction of S0s in the groups has occured to be at least as high as in $z \sim 0.4$ clusters and much higher than in the field at $z \sim 0.4$. So the groups now look more promising as the site of S0 galaxies formation at intermediate redshifts, and the clusters acquired probably the fully shaped S0s with accreted groups. Then the hydrodynamical mechanisms related to the hot intergalactic medium cannot play the major role in spiral morphological transformation: in groups which only in half cases possess X-ray gas the gravitational mechanisms such as galaxy-galaxy interaction and minor merging are the main players. Just et al. [52] investigated the role of dynamical characteristics of the environment in the S0 fraction evolution between $z=0.8$ and $z=0$; the groups and clusters were probed. The result was that the S0 fraction changes dramatically between $z=0.4$ and $z=0$ in the groups and poor clusters with the galaxy velocity dispersion less than $750 \mathrm{~km} / \mathrm{s}$; in rich massive clusters the evolution was not so strong. This discovery constrains further the plausible mechanisms of S0 formation if it takes place at $z \sim 0.4$ : the gravitational interaction between a galaxy and an environment (e.g., cluster dark halo potential) seems to be less important than pairwise galaxy interactions.

Based on these observational results, Bekki and Couch [53] have developed a scenario of spiral galaxy transformation into S0 within a group environment. Indeed, galaxy-galaxy slow-speed encounters have occurred to be quite effective as concerning the necessary morphological changes: after 5.6 Gyr of numerical evolution, the spiral galaxy turns into S0, with the dynamically hot thick stellar disk, without spiral arms or noticeable gas content, and with some young stars concentrated in the very center. The simulations reveal repetitive starbursts provoked by tidal interaction with other galaxies which are confined to the central part of the galaxy and which increase significantly the mass of the galaxy bulge during this morphological transformation. So the lenticular galaxies "produced" within the Bekki and Couch's scenario must have rather young, metal-rich bulges.

\subsection{Our New Ideas}

However, all these scenaria relate to dense environments. Meantime there are a lot of S0s in the field in the nearby Universe, they constitute $15 \%$ of all galaxies locally, being at the second place after spirals [54] - much more in absolute terms than S0s in nearby clusters. Moreover, there is a population of strictly isolated S0s [38]. How can they be formed?

We can also point out that the strict epoch of forming S0s in clusters, $z=0.4$, found by studying intermediate-redshift clusters (see above), is completely discarded by the observations of stellar populations in the disks of nearby S0s: they are mostly old, older than $10 \mathrm{Gyr}$, and the denser are their environments the older are S0 stellar disks on average $[55,56]$. We can conclude that nearby S0s in dense enviroments had completely shaped their disks at $z>1.5$ and do not relate at all to the Butcher-Oemler effect. The mechanisms to form thick passive stellar disks at $z>1.5$ may be various: they can be formed by vigorous star formation in the clumpy turbulent gas-dominated galaxies observed at these redshifts [57-59] or by major merging of gas-rich disk galaxies—e.g., [60]—but the firm conclusion is that S0 galaxies are the primary population of disk galaxies. Between $z=1.5$ and $z=1$ perhaps all disk galaxies were S0s, including our own Galaxy [61].

As we have reviewed in the Introduction, the most lenticular galaxies contain cold gas, sometimes rather abundant. However, the morphological type of S0 galaxies is characterized by the 
absence of noticeable star formation in their disks, by the definition of $S 0$ type. Pogge and Eskridge who searched for star formation in HI-rich S0 galaxies [62], found HII-regions only in the half of the disks of the lenticulars with a large amount of neutral hydrogen. Their final conclusion made after the study of the full sample of 32 gas-rich S0s [63] was that, firstly, star formation in the lenticulars was always organized in ring-like structures, not in spirals, and secondly, the occurence of star formation where it was found did not depend on the amount of gas available. A hypothesis was then formulated in [63] that star formation in gas-rich S0s had to be drived not by intrinsic gravitational instability, as in spiral galaxies, but, taking into account its threshold nature, by some external kinematical trigger.

Now we know, or at least we are sure, that all spiral galaxies experience continuous outer metal-poor gas accretion providing the continuous star formation in their disks-it is a necessary condition of their evolution due to, firstly, the absence of visible metal enrichment of the disk stellar populations during the last $8 \mathrm{Gyr}$ (so called "G-dwarf paradox", see for example [2,3,64]), and secondly, due to the very short gas depletion time in nearby spiral galaxies (see for example [4]). Lenticular galaxies are also disk galaxies, and they may also always experience gas accretion, at least when they reside in sparse environments. What conditions may separate spirals and lenticulars as concerning their gas supply? Why is there intense star formation in spirals and no intense star formation in lenticulars, despite their gas content? Haynes et al. [65] compared mean gas surface density in lenticulars and early spirals and found that the ranges of $\sigma_{\mathrm{HI}}$ are the same in Sa and in $\mathrm{S} 0$ in low-density environments. So if we appeal only to the Kennicutt-Schmidt law, the star formation would be the same in S0's and Sa's; however, it is not so. There exists another factor which distinguishes S0s from spirals, and it may be a temperature of the gas and the geometry of accretion.

I can propose the following explanation for a variety of evolutionary paths of disk galaxies. All disk galaxies experience outer gas accretion, the source of which is beyond our discussion-it may be cosmological filaments or satellite systems, taking into account that the most active past accretors are presently quite isolated galaxies. If the gas accretion is confined to the main galactic plane, is abundant and laminar-the galaxy becomes a spiral one at rather recent epoch, $z<1$, when (or where) we start to observe thin stellar disks. If the accretion is poor, intermittent, or from the direction inclined to the main plane of the galactic disk-the galaxy remains a lenticular one. Curious findings have been reported in [66]: among rather isolated galaxies, the early-type ones, mostly lenticular galaxies have no satellites with the LOS velocity difference between the satellite and the host of less than $50 \mathrm{~km} / \mathrm{s}$, while isolated spiral galaxies have a lot of such satellites. This may be direct evidence for a scarcer (on average) resource of satellite accretion corresponding to earlier galaxy morphology. However, even intense accretion from an inclined direction may heat the gas when passing through the potential well of the galactic disk, and this heating may prevent the subsequent star formation in the accreted gaseous disk.

Acknowledgments: I thank my colleagues Alexei Moiseev and Victor Afanasiev having supported the spectral part of the project fulfilled at the Russian $6 \mathrm{~m}$ telescope financed by the Ministry of Science and Education of the Russian Federation (agreement no.14.619.21.0004, project identificator RFMEFI61914X0004); many ideas, calculations, and publications have been shared by the author with Ivan Katkov. The present study makes use of data obtained from the Isaac Newton Group Archive which is maintained as a part of the CASU Astronomical Data Centre at the Institute of Astronomy, Cambridge.

Conflicts of Interest: The author declares no conflict of interest.

\section{References}

1. Kaviraj, S.; Peirani, S.; Khochfar, S.; Silk, J.; Kay, S. The role of minor mergers in the recent star formation history of early-type galaxies. Mon. Not. R. Astron. Soc. 2009, 394, 1713-1720.

2. Tosi, M. The effect of metal-rich infall on galactic chemical evolution. Astron. Astrophys. 1988, 197, 47-51.

3. Chiosi, C. Chemical evolution of the galactic disk - The inflow problem. Astron. Astrophys. 1980, 83, 206-216. 
4. Bigiel, F.; Leroy, A.K.; Walter, F.; Brinks, E.; de Blok, W.J.G.; Kramer, C.; Rix, H.W.; Schruba, A.; Schuster, K.F.; Usero, A.; et al. A Constant Molecular Gas Depletion Time in Nearby Disk Galaxies. Astrophys. J. Lett. 2011, 730, L13,

5. Welch, G.A.; Sage, L.J.; Young, L.M. The Cool Interstellar Medium in Elliptical Galaxies. II. Gas Content in the Volume-limited Sample and Results from the Combined Elliptical and Lenticular Surveys. Astrophys. J. 2010, 725, 100-114.

6. Serra, P.; Oosterloo, T.; Morganti, R.; Alatalo, K.; Blitz, L.; Bois, M.; Bournaud, F.; Bureau, M.; Cappellari, M.; et al. The ATLAS ${ }^{3 D}$ project - XIII. Mass and morphology of H I in early-type galaxies as a function of environment. Mon. Not. R. Astron. Soc. 2012, 422, 1835-1862.

7. Davis, T.A.; Alatalo, K.; Sarzi, M.; Bureau, M.; Young, L.M.; Blitz, L.; Serra, P.; Crocker, A.F.; Krajnović, D.; McDermid, R.M.; et al. The ATLAS ${ }^{3 D}$ project $-X$. On the origin of the molecular and ionized gas in early-type galaxies. Mon. Not. R. Astron. Soc. 2011, 417, 882-899.

8. Sarzi, M.; Shields, J.C.; Schawinski, K.; Jeong, H.; Shapiro, K.; Bacon, R.; Bureau, M.; Cappellari, M.; Davies, R.L.; de Zeeuw, P.T.; et al. The SAURON project - XVI. On the sources of ionization for the gas in elliptical and lenticular galaxies. Mon. Not. R. Astron. Soc. 2010, 402, 2187-2210.

9. Bertola, F.; Buson, L.M.; Zeilinger, W.W. The external origin of the gas in S0 galaxies. Astrophys. J. 1992, 401, L79-L81.

10. Kuijken, K.; Fisher, D.; Merrifield, M.R. A search for counter-rotating stars in S0 galaxies. Mon. Not. R. Astron. Soc. 1996, 283, 543-550.

11. Katkov, I.Y.; Sil'chenko, O.K.; Afanasiev, V.L. Decoupled gas kinematics in isolated S0 galaxies. Mon. Not. R. Astron. Soc. 2014, 438, 2798-2803.

12. Bettoni, D.; Fasano, G.; Galletta, G. NGC 2217, a case of apparent counterrotation. Astron. J. 1990, 99, 1789-1797.

13. Sil'chenko, O.K.; Vlasyuk, V.V.; Burenkov, A.N. Decoupled nuclei and nuclear polar rings in regular spiral galaxies. NGC 2841. Astron. Astrophys. 1997, 326, 941-949.

14. Sil'chenko, O.K.; Afanasiev, V.L. Decoupled nuclei and nuclear polar rings in regular spiral galaxies. NGC 7217. Astron. Astrophys. 2000, 364, 479-490.

15. Moiseev, A.V. Inner polar rings and disks: Observed properties. Astrophys. Bull. 2012, 67, 147-159.

16. Corsini, E.M.; Pizzella, A.; Coccato, L.; Bertola, F. Minor-axis velocity gradients in spirals and the case of inner polar disks. Astron. Astrophys. 2003, 408, 873-885.

17. Sil'chenko, O.K.; Afanasiev, V.L. Inner Polar Rings in Regular Lenticular Galaxies. Astron. J. 2004, 127, 2641-2658.

18. Cappellari, M.; Emsellem, E.; Krajnović, D.; McDermid, R.M.; Scott, N.; Verdoes Kleijn, G.A.; Young, L.M.; Alatalo, K.; Bacon, R.; Blitz, L.; et al. The ATLAS ${ }^{3 D}$ project - I. A volume-limited sample of 260 nearby early-type galaxies: Science goals and selection criteria. Mon. Not. R. Astron. Soc. 2011, 413, 813-836.

19. Reshetnikov, V.P.; Faúndez-Abans, M.; de Oliveira-Abans, M. Polar-ring galaxies: New candidates and statistics. Astron. Lett. 2011, 37, 171-180.

20. Moiseev, A.V.; Smirnova, K.I.; Smirnova, A.A.; Reshetnikov, V.P. A new catalogue of polar-ring galaxies selected from the Sloan Digital Sky Survey. Mon. Not. R. Astron. Soc. 2011, 418, 244-257.

21. Afanasiev, V.L.; Sil'chenko, O.K. Young Stellar Nuclei in the Lenticular Galaxies. II. NGC 7280. Astron. J. 2000, 119, 126-135.

22. Van Albada, T.S.; Kotanyi, C.G.; Schwarzschild, M. A model for elliptical radio galaxies with dust lanes. Mon. Not. R. Astron. Soc. 1982, 198, 303-310.

23. Friedli, D.; Benz, W. Secular evolution of isolated barred galaxies. I - Gravitational coupling between stellar bars and interstellar medium. Astron. Astrophys. 1993, 268, 65-85.

24. Sil'chenko, O.K. Chemically Decoupled Nuclei in Five Lenticular Galaxies from SAURON Data. Astron. Lett. 2005, 31, 227-238.

25. Sil'chenko, O.K.; Moiseev, A.V. Nature of Nuclear Rings in Unbarred Galaxies: NGC 7742 and NGC 7217. Astron. J. 2006, 131, 1336-1346.

26. Chilingarian, I.V.; di Matteo, P.; Combes, F.; Melchior, A.L.; Semelin, B. The GalMer database: Galaxy mergers in the virtual observatory. Astron. Astrophys. 2010, 518, A61.

27. Sil'chenko, O.K.; Chilingarian, I.V.; Sotnikova, N.Y.; Afanasiev, V.L. Large-scale nested stellar discs in NGC 7217. Mon. Not. R. Astron. Soc. 2011, 414, 3645-3655. 
28. Van de Voort, F.; Davis, T.A.; Kereš, D.; Quataert, E.; Faucher-Giguère, C.A.; Hopkins, P.F. The creation and persistence of a misaligned gas disc in a simulated early-type galaxy. Mon. Not. R. Astron. Soc. 2015, 451, 3269-3277.

29. Katkov, I.Y.; Moiseev, A.V.; Sil'chenko, O.K. Stars and Ionized Gas in the S0 Galaxy NGC 7743: An Inclined Large-scale Gaseous Disk. Astrophys. J. 2011, 740, 83.

30. Sil'chenko, O.K.; Moiseev, A.V.; Afanasiev, V.L. Two More Disk Galaxies with Global Gas Counterrotation. Astrophys. J. 2009, 694, 1550-1558.

31. Bertola, F.; Cinzano, P.; Corsini, E.M.; Pizzella, A.; Persic, M.; Salucci, P. Counterrotating Stellar Disks in Early-Type Spirals: NGC 3593. Astrophys. J. 1996, 458, L67.

32. Jore, K.P.; Broeils, A.H.; Haynes, M.P. A Counter-Rotating Disk in the Normal SA Galaxy NGC 4138. Astron. J. 1996, 112, 438-456.

33. Coccato, L.; Morelli, L.; Corsini, E.M.; Buson, L.; Pizzella, A.; Vergani, D.; Bertola, F. Dating the formation of the counter-rotating stellar disc in the spiral galaxy NGC 5719 by disentangling its stellar populations. Mon. Not. R. Astron. Soc. 2011, 412, L113-L117.

34. Katkov, I.Y.; Sil'chenko, O.K.; Afanasiev, V.L. Lenticular Galaxy IC 719: Current Building of the Counterrotating Large-scale Stellar Disk. Astrophys. J. 2013, 769, 105.

35. Afanasiev, V.L.; Moiseev, A.V. Scorpio on the $6 \mathrm{~m}$ Telescope: Current State and Perspectives for Spectroscopy of Galactic and Extragalactic Objects. Baltic Astron. 2011, 20, 363-370.

36. Krajnović, D.; Emsellem, E.; Cappellari, M.; Alatalo, K.; Blitz, L.; Bois, M.; Bournaud, F.; Bureau, M.; Davies, R.L.; Davis, T.A.; et al. The ATLAS ${ }^{3 D}$ project - II. Morphologies, kinemetric features and alignment between photometric and kinematic axes of early-type galaxies. Mon. Not. R. Astron. Soc. 2011, 414, 2923-2949.

37. Coccato, L.; Fabricius, M.; Morelli, L.; Corsini, E.M.; Pizzella, A.; Erwin, P.; Dalla Bontà, E.; Saglia, R.; Bender, R.; Williams, M. Properties and formation mechanism of the stellar counter-rotating components in NGC 4191. Astron. Astrophys. 2015, 581, A65.

38. Katkov, I.Y.; Kniazev, A.Y.; Sil'chenko, O.K. Kinematics and Stellar Populations in Isolated Lenticular Galaxies. Astron. J. 2015, 150, 24.

39. Butcher, H.; Oemler, Jr., A. The evolution of galaxies in clusters. V - A study of populations since Z approximately equal to 0.5 . Astrophys. J. 1984, 285, 426-438.

40. Dressler, A.; Oemler, A., Jr.; Couch, W.J.; Smail, I.; Ellis, R.S.; Barger, A.; Butcher, H.; Poggianti, B.M.; Sharples, R.M. Evolution since $\mathrm{z}=0.5$ of the Morphology-Density Relation for Clusters of Galaxies. Astrophys. J. 1997, 490, 577-591.

41. Fasano, G.; Poggianti, B.M.; Couch, W.J.; Bettoni, D.; Kjærgaard, P.; Moles, M. The Evolution of the Galactic Morphological Types in Clusters. Astrophys. J. 2000, 542, 673-683.

42. Larson, R.B.; Tinsley, B.M.; Caldwell, C.N. The evolution of disk galaxies and the origin of S0 galaxies. Astrophys. J. 1980, 237, 692-707.

43. Bekki, K.; Couch, W.J.; Shioya, Y. Passive Spiral Formation from Halo Gas Starvation: Gradual Transformation into S0s. Astrophys. J. 2002, 577, 651-657.

44. Van den Bergh, S. Lenticular Galaxies and their Environments. Astrophys. J. 2009, 702, 1502-1506.

45. Gunn, J.E.; Gott, III, J.R. On the Infall of Matter Into Clusters of Galaxies and Some Effects on Their Evolution. Astrophys. J. 1972, 176, 1-19.

46. Quilis, V.; Moore, B.; Bower, R. Gone with the Wind: The Origin of S0 Galaxies in Clusters. Science 2000, $288,1617-1620$.

47. Kronberger, T.; Kapferer, W.; Ferrari, C.; Unterguggenberger, S.; Schindler, S. On the influence of ram-pressure stripping on the star formation of simulated spiral galaxies. Astron. Astrophys. 2008, 481, 337-343.

48. Moore, B.; Katz, N.; Lake, G.; Dressler, A.; Oemler, A. Galaxy harassment and the evolution of clusters of galaxies. Nature 1996, 379, 613-616.

49. Icke, V. Distant encounters between disk galaxies and the origin of S0 spirals. Astron. Astrophys. 1985, 144, 115-123.

50. Byrd, G.; Valtonen, M. Tidal generation of active spirals and S0 galaxies by rich clusters. Astrophys. J. 1990, 350, 89-94. 
51. Wilman, D.J.; Oemler, Jr., A.; Mulchaey, J.S.; McGee, S.L.; Balogh, M.L.; Bower, R.G. Morphological Composition of $\mathrm{z} \sim 0.4$ Groups: The Site of S0 Formation. Astrophys. J. 2009, 692, 298-308.

52. Just, D.W.; Zaritsky, D.; Sand, D.J.; Desai, V.; Rudnick, G. The Environmental Dependence of the Evolving S0 Fraction. Astrophys. J. 2010, 711, 192-200.

53. Bekki, K.; Couch, W.J. Transformation from spirals into S0s with bulge growth in groups of galaxies. Mon. Not. R. Astron. Soc. 2011, 415, 1783-1796.

54. Naim, A.; Lahav, O.; Buta, R.J.; Corwin, Jr., H.G.; de Vaucouleurs, G.; Dressler, A.; Huchra, J.P.; van den Bergh, S.; Raychaudhury, S.; Sodre, L., Jr.; Storrie-Lombardi, M.C. A comparative study of morphological classifications of APM galaxies. Mon. Not. R. Astron. Soc. 1995, 274, 1107-1125.

55. Sil'chenko, O.K.; Proshina, I.S.; Shulga, A.P.; Koposov, S.E. Ages and abundances in large-scale stellar discs of nearby S0 galaxies. Mon. Not. R. Astron. Soc. 2012, 427, 790-805.

56. Johnston, E.J.; Aragón-Salamanca, A.; Merrifield, M.R. The origin of S0s in clusters: Evidence from the bulge and disc star formation histories. Mon. Not. R. Astron. Soc. 2014, 441, 333-342.

57. Elmegreen, D.M.; Elmegreen, B.G.; Rubin, D.S.; Schaffer, M.A. Galaxy Morphologies in the Hubble Ultra Deep Field: Dominance of Linear Structures at the Detection Limit. Astrophys. J. 2005, 631, 85-100.

58. Bournaud, F.; Elmegreen, B.G.; Elmegreen, D.M. Rapid Formation of Exponential Disks and Bulges at High Redshift from the Dynamical Evolution of Clump-Cluster and Chain Galaxies. Astrophys. J. 2007, $670,237-248$.

59. Bournaud, F.; Elmegreen, B.G. Unstable Disks at High Redshift: Evidence for Smooth Accretion in Galaxy Formation. Astrophys. J. Lett. 2009, 694, L158-L161.

60. Querejeta, M.; Eliche-Moral, M.C.; Tapia, T.; Borlaff, A.; van de Ven, G.; Lyubenova, M.; Martig, M.; Falcón-Barroso, J.; Méndez-Abreu, J. Formation of S0 galaxies through mergers. Explaining angular momentum and concentration change from spirals to S0s. Astron. Astrophys. 2015, 579, L2.

61. Fuhrmann, K. The Disk Populations in the [Mg/H]-[Fe/Mg] Plane. Astrophys. Space Sci. 1999, 265, $265-268$.

62. Pogge, R.W.; Eskridge, P.B. Star-forming regions in gas-rich lenticulars. I. H-alpha imaging of an initial sample of galaxies. Astron. J. 1987, 93, 291-300.

63. Pogge, R.W.; Eskridge, P.B. Star formation in the disks of H I-rich S0 galaxies. Astron. J. 1993, 106, $1405-1419$.

64. Dalcanton, J.J. The Metallicity of Galaxy Disks: Infall versus Outflow. Astrophys. J. 2007, 658, 941-959.

65. Haynes, M.P.; Herter, T.; Barton, A.S.; Benensohn, J.S. The influence of environment on gas and dust in S0 galaxies. Astron. J. 1990, 99, 1740-1772.

66. Karachentseva, V.E.; Karachentsev, I.D.; Melnyk, O.V. Faint companions of isolated 2MIG galaxies. Astrophys. Bull. 2011, 66, 389-406.

(C) 2015 by the authors; licensee MDPI, Basel, Switzerland. This article is an open access article distributed under the terms and conditions of the Creative Commons by Attribution (CC-BY) license (http://creativecommons.org/licenses/by/4.0/). 